\title{
PEQUENOS NEGÓCIOS E FLUXO DE INFORMAÇÕES EM COMUNIDADE DE IMIGRANTES: ENSAIO SOBRE APLICAÇÃO DA NETNOGRAFIA
}

\author{
SMALL BUSINESS AND INFORMATION FLOW IN THE IMMIGRANT \\ COMMUNITY: ESSAY ON APPLICATION OF NETNOGRAPHY
}

Recebido em 23.02.2018. Aprovado em 29.04.2018 Avaliado pelo sistema double blind review

Roberto Pessoa de Queiroz Falcão

robertopgfalcao@gmail.com

Pontifícia Universidade Católica do Rio de Janeiro (IAG/PUC-Rio) - Rio de Janeiro - RJ

Andrea Ribeiro Carvalho Castro

andrearc.castro@gmail.com

Pontifícia Universidade Católica do Rio de Janeiro (IAG/PUC-Rio) - Rio de Janeiro - RJ

Eduardo Picanço Cruz

epicanco@id.uff.br

Universidade Federal Fluminense - Departamento de Empreendedorismo e Gestão - Rio de Janeiro - RJ

Sabrina Sampaio Amaral

sabrina_sampaio@id.uff.br

Universidade Federal Fluminense - Departamento de Empreendedorismo e Gestão - Rio de Janeiro - RJ

\begin{abstract}
Resumo
O presente artigo discute a aplicação da netnografia, ou etnografia de comunidades online, na investigação de atividades empreendedoras e fluxos de informação, que acontecem em comunidades de imigrantes no exterior. Os autores apresentam o método e discutem sua aplicação no contexto de uma das comunidades do Facebook, de imigrantes brasileiros em Sidney, Austrália. Após a coleta de dados de postagens através da netnografia, utilizou-se um software de análise de conteúdo para facilitar a codificação de alguns comportamentos do comércio informal e do fluxo de informações que se configura neste ambiente online. 0 estudo exploratório identificou que a metodologia é adequada e promissora para este contexto. Os achados relativos à comunidade pesquisada, incluem aspectos utilitários de fluxos de informação e venda de bens e serviços, assim como dimensões simbólicas, relativas ao pertencimento dos membros da comunidade.
\end{abstract}

Palavras-chaves: Netnografia; Empreendedorismo; Imigrante.

\section{Abstract}

This article discusses the application of netnography, or ethnography of online communities, in the investigation of entrepreneurial activities and information flows, that take place in communities of immigrants overseas. The authors present the method and discuss its application in the context of one of the Facebook communities of Brazilian immigrants in Sydney, Australia. After collecting posts and data through netnography, a content analysis software was used to facilitate the coding of some behaviors of informal commerce and the flow of informations that is configured in this online environment. The exploratory study identified that the methodology is adequate and promising for this context. The findings related to the 
community surveyed include utilitarian aspects of information flows and sales of goods and services, as well as symbolic dimensions related to the sense of belonging of community.

Keywords: Ethnography; Entrepreneurial; Immigrants.

\section{Introdução}

O estudo de diferentes tipos de comunidades tornou-se um tópico importante no campo do comportamento de consumo (LUEDICKE, 2006; MCALEXANDER; SCHOUTEN; KOENIG, 2002; SCHAU; MUÑIZ; ARNOULD, 2009; ARNOULD; THOMPSON, 2005). Na última década, no entanto, houve um crescimento no interesse em se investigar as atividades de consumo em comunidades online (COVA; PACE, 2006; KOZINETS, 2002).

A ascensão da sociedade de rede, proclamada há mais de 20 anos por Castells (1996), foi consolidada justamente neste mesmo período, com o crescimento da internet, a globalização dos fluxos de informação, e o comércio de produtos e serviços através dos meios digitais (APPADURAI, 1990; BENNETT, 1999; KJELDGAARD; ASKEGAARD, 2006). Ambientes online contemporâneos são reconhecidos como catalisadores para a fragmentação de mercados, o que corrobora com o panorama multifacetado do consumo "pós-moderno" (BROWN, 1995; FIRAT; DHOLAKIA, 2006; FIRAT; VENKATESH, 1995). Por conseguinte, as comunidades presentes nos ambientes online, tornaram-se desprendidas de localização. Seus membros podem ser indivíduos que compartilham várias conexões através de tablets, computadores e celulares, mantendo sua presença digital simultaneamente em diversas comunidades globais (KOZINETS, 2014).

Por outro lado, comunidades de imigrantes estabelecidas em diversos países se engajam em interações frequentes tanto no mundo real quanto no mundo virtual. Essas interações podem ser ligadas a negócios ou fluxo de informação. São exemplos da primeira categoria: venda de produtos e serviços do país de origem, aluguel de quartos para compatriotas, ou ofertas de emprego. Já no contexto dos fluxos de informação, os membros das comunidades de imigrantes podem demandar dicas de lugares, pedir ajuda, doar itens, ou simplesmente dar informações de utilidade pública a respeito de saúde, vistos ou assuntos gerais. Relativo aos estudos acadêmicos de empreendedorismo imigrante, alguns autores destacam-se no campo, tais como Waldinger, Aldrich e Ward (1990), Butler (2001), Barrett, Jones e McEvoy (1996), Rath e Kloosterman (2000), Drori, Honig e Wright (2009), Achidi e Priem (2011), Portes e Zhou (1992), Zhou (2004), Chrysostome e Lin (2010). Em recentes revisões bibliométricas do tema de empreendedorismo imigrante (MA et al., 2013; ALIAGA-ISLA; RIALP, 2013) não foram encontrados artigos a respeito do comércio e do fluxo de informações de comunidades imigrantes no meio online. Há, portanto, uma lacuna de pesquisa na interseção entre o comportamento de consumo e o empreendedorismo de imigrantes.

Segundo Aliaga-Isla e Rialp (2013), diversos estudos relativos ao empreendedorismo imigrante se utilizam de abordagens qualitativas, coletando dados através de entrevistas semi-estruturadas, telefone, e-mail, website, snowball, dentre outros (VAN DELFT; GORTER; NIJKAMP, 2000; ESSERS; BENSCHOP; DOOREWAARD, 2010; KIM; HURH,1985; MIERA, 2008; TEIXEIRA; LO; TRUELOVE, 2007). Contudo, não foram encontrados, artigos que seguissem a metodologia de netnografia para se avaliar comunidades imigrantes, o que se apresenta como lacuna da aplicação desta metodologia.

Relativo ao método empregado no presente artigo - a netnografia, o mesmo foi desenvolvido por Kozinets (2002) como uma técnica de pesquisa de marketing online, para obtenção de insights do consumidor. Ela seria 0 equivalente da etnografia, adaptado ao contexto do estudo das comunidades online. 0 método apresenta algumas vantagens quando comparado ao seu original. É de aplicação mais rápida, mais simples e menos dispendiosa do que a etnografia tradicional. Além disso, segundo afirma seu criador, ela seria mais naturalística e discreta se comparada aos grupos focais ou entrevistas em profundidade.

Referente à sua aplicação, no contexto da pesquisa dos pequenos negócios de imigrantes, a técnica pode 
fornecer informações sobre o simbolismo, pertencimento, os significados e práticas de consumidores e vendedores online. As informações coletadas podem ser também de cunho não comercial, embora não menos relevantes para o entendimento dessas comunidades. Em seus artigos seminais, Kozinets (1998, 1999) ainda fornece um protocolo de pesquisa a ser seguido com rigor e ética.

Assim, o objetivo desse artigo foi o de analisar a aplicação do método em uma das comunidades do Facebook de imigrantes brasileiros no exterior - Brasileiros em Sydney. Os autores relatam o protocolo de pesquisa, vantagens, desvantagens de sua aplicação, dificuldades na condução e oportunidades de sua aplicação no contexto do empreendedorismo, do estudo de mercados e comportamento de consumo de comunidades online. Através da netnografia, buscou-se codificar alguns comportamentos do comércio informal que se configuram nestes ambientes online, assim como do fluxo de informações. $O$ estudo exploratório do método aplicado à comunidade virtual de imigrantes se mostrou promissor, pois foi capaz de evidenciar dimensões importante do senso de comunidade, comportamentos relativos a negócios e fluxos de informação. Além disso, identificou caminhos para pesquisa a respeito da importância dos produtos e serviços vendidos através dessa plataforma, assim como o entendimento a respeito da forma como se configuram esses fluxos, sua importância para a sobrevivência e para o pertencimento dos membros da comunidade.

\section{Fundamentação}

\section{A Diáspora Brasileira}

Butler (2001) identifica vários tipos de diáspora, baseando-se nos motivos por trás de sua formação, por exemplo, cativeiro, exílio, emigração, etc. De acordo com este autor, há consenso entre os estudiosos de que três condições são necessárias para definir uma diáspora. Primeiro, as pessoas devem estar espalhadas em pelo menos dois países de acolhimento. Em segundo lugar, deve haver uma relação contínua com a pátria (real ou idealizada). Em terceiro lugar, as pessoas devem mostrar autoconsciência de sua identidade de grupo. $\mathrm{O}$ autor ainda acrescenta uma quarta condição, de que 0 processo inclua pelo menos duas gerações.

Uma diáspora pode ser entendida, portanto, como uma "rede sócioespacial" ancorada num capital simbólico que the permite reproduzir e superar o obstáculo muitas vezes considerável da distância que separa suas comunidades (BRUNEAU, 2010, p.36).

PRIES (2004) descreve quatro tipos de migrantes internacionais: (a) o emigrante/imigrante, que tem a intenção de adotar o país de destino como sua nova pátria; (b) o migrante de retorno, que pretende regressar à sua terra natal em um curto período de tempo; (c) o migrante de diáspora, que mantém a ligação com a pátria, e evita a integração; e (d) o transmigrante, que é ambíguo sobre a natureza e duração de sua migração.

O Brasil tradicionalmente é um país receptor de imigrantes, sendo a diáspora brasileira um fenômeno mais recente, alavancada por sucessivas crises econômicas. Segundo Martes, Rodriguez (2004) e Margolis (1995), as ondas migratórias se intensificaram na década de 1980, sob um cenário de hiperinflação. Muitos brasileiros vieram a se estabelecer nos Estados Unidos, em cidades como Boston, Nova lorque, Miami e Orlando. Brasileiros de origem japonesa, intitulados decasséguis, partiram em movimento migratório circular para trabalhar nos anos 1980 e 1990 nas fabricas japonesas, juntando dinheiro para posteriormente montarem seus negócios ou comprarem terras no Brasil (SASAKI, 1999, 2006). Muitos brasileiros de origem europeia com ancestralidade italiana, portuguesa, espanhola e alemã, solicitaram seus passaportes europeus para posteriormente imigrarem. Em paralelo após os anos 1990 o mundo também ficou mais global para os brasileiros que encorparam as massas de estudantes estrangeiros, em programas de ensino de idioma e intercâmbio no Canadá, Estados Unidos, Europa e Austrália. Recentemente o programa governamental "Ciências sem Fronteiras" também contribuiu para proporcionar mais experiências internacionais a estudantes e pesquisadores. Segundo Nkongolo-Bakenda e Chrysostome (2013), a 
pesquisa da diáspora no campo do empreendedorismo internacional ainda está em seu início, o que corrobora com a lacuna teórico-empírica encontrada.

\section{Negócios de Imigrantes e Senso de Comunidade Online e Off-Line}

Como o objetivo deste trabalho é o de discutir a adaptação do método da netnografia no contexto das comunidades imigrantes no que diz respeito às suas atividades empreendedoras e fluxo de informações, é importante apresentar algumas das principais abordagens e conceitos.

No que diz respeito ao empreendedorismo, os agrupamentos de imigrantes em áreas geográficas comuns permitem a formação de enclaves étnicos, onde é evidenciado o comércio de produtos típicos da nacionalidade, ofertas de empregos para compatriotas, redes específicas de fornecedores clientes e circulação de capital. Segundo Zhou (2004) são negócios culturalmente unidos, que mantém laços de solidariedade e confiança. Além disso, são capazes de aumentar oportunidades econômicas e formar redes de proteção que alavancam recursos para usufruto de empreendimentos imigrantes (DRORI; HONIG; WRIGHT, 2009).

Empresários transnacionais são caracterizados como aqueles que estabelecem vínculos comerciais no país que os hospeda, assim como em sua terra natal (BALTAR; ICART, 2013). Os enclaves étnicos também dão suporte à criação de pequenos e médios negócios transnacionais, uma vez que se configuram como redes de negócios que oferecem conexões com outros empresários locais. Segundo Drori, Honig e Wright (2009) ambos os empresários "transnacional" e "étnicos", têm capacidade de tirar proveito de alavancagens culturais de seu próprio capital social.

O crescimento do "empreendedorismo transnacional" nos últimos anos deveu-se a fatores como a natureza da constante mudança da migração internacional (LIGHT, 2007; RIDDLE, 2008); e a prevalência de serviços de telecomunicação, e-mail, internet e viagens aéreas cada vez mais acessíveis (DRORI; HONI; WRIGHT, 2009).

No tocante ao fluxo de informação, as redes sociais assumem um papel misto de tecnologia de informação e veículo de mídia, mantendo os laços dos imigrantes com os países de origem. Além disso, são fontes de conteúdo, de comércio e de manifestações significativas de capital social (NAVARRETE; HUERTA, 2006). De acordo com Fisher, Sonn e Bishop (2002) o apego emocional, que fornece laços de união dessas comunidades, é baseado em histórias compartilhadas, experiências comuns, que podem se manifestar nos diferentes tipos de comunidade no mundo físico (por exemplo, eventos sociais e culturais, clubes desportivos e igrejas).

Estudos recentes sobre comunidades virtuais adotaram a definição de Blanchard e Markus (2004), baseada no trabalho de McMillan e Chavis (1986) para diferenciar os dois tipos de 'senso de comunidade' [SOC]: os que surgem de comunidades físicas e os oriundos de comunidades virtuais - o 'senso de comunidade virtual' [SOVC]. Assim como em um mundo físico, que nem todos os assentamentos desenvolvem um SOC, também no mundo virtual, nem todos os assentamentos virtuais conseguem fomentar um SOVC.

A SOVC tem sido investigada em diferentes contextos. Blanchard e Markus (2004) identificaram quatro dimensões da SOC que podem ser evidenciadas empiricamente nas comunidades virtuais: 'adesão', 'influência', 'integração e realização' e 'conexão emocional compartilhada'. Navarrete e Huerta (2006) citam que Blanchard e Markus (2004) encontraram evidências de mais duas dimensões ao modelo: 'identidade e identificação' e 'relacionamento com membros específicos'.

A dimensão 'adesão' refere-se ao sentimento de pertencimento ou compartilhamento de um sentimento de parentesco pessoal. Envolve ainda os sistemas de símbolos que tenham um significado particular para a comunidade, como a linguagem, valores culturais, gostos, alimentação e vestuário, e uma história de desafios e batalhas comuns (MCMILLAN; CHAVIS, 1986). 'Influência' requer uma abordagem bidirecional, onde os membros podem influenciar o grupo ou serem atraídos pelo mesmo. 'Integração e satisfação das 
necessidades' são observados nas experiências vividas como, por exemplo: a forma de enviar dinheiro para sua terra natal, onde encontrar determinados ingredientes para seus pratos típicos, ou locais de moradia (NAVARRETE; HUERTA, 2006).

A última dimensão da SOVC é a 'conexão emocional compartilhada', que pode ser caracterizada como os compromissos e crenças as quais os membros compartilham, tais como 'histórias, lugares comuns, experiências e eventos semelhantes', muito presente na vida de um imigrante.

Como mencionado anteriormente, as dimensões adicionadas por Blanchard e Markus (2004) identidade e identificação, e relacionamento com membros específicos, também geram influências sobre os membros das comunidades. Os autores argumentam que a identificação com a comunidade descrita por McMillan e Chavis (1986) se refere ao sentimento de compartilhar a identidade do grupo. Ou seja, um imigrante se identificará com outros imigrantes. Ser um imigrante será o passo inicial para pertencer à comunidade. No entanto, nas comunidades virtuais puras, falta a interação face a face, portanto, os membros da comunidade virtual sentem a necessidade de identificação individual dentro do grupo. Uma vez que os membros das comunidades virtuais mantêm relações físicas e virtuais simultaneamente, este senso de identificação será diferente das comunidades virtuais puras. A comunidade virtual extrapola as identificações da comunidade física. Blanchard e Markus (2004) identificaram várias técnicas de identificação, tais como formas de assinatura ou "uma citação espirituosa ou trocadilho no final de uma postagem". Como, as restrições de comunicação são iguais tanto em comunidades virtuais de imigrantes como em qualquer outro tipo de comunidade virtual, pode-se esperar que os membros de comunidades de imigrantes usem técnicas de identificação semelhantes.

Finalmente, a última dimensão é o relacionamento ou "relação" entre membros específicos, refere-se ao desenvolvimento de relações que se alternam entre virtual e face a face. Em uma comunidade virtual de imigrantes normalmente as relações coexistem online e off-line. Os membros potenciais podem se conhecer das comunidades virtuais ou de relações face a face. A dimensão da relação impacta o processo de "criação de confiança".

\section{Considerações sobre a Netnografia}

A netnografia está inserida na categoria de métodos qualitativos, os quais são particularmente úteis para revelar detalhes de riqueza simbólica subjacente às necessidades, desejos, significados e escolhas dos consumidores (DENZIN; LINCOLN, 1994). Os métodos qualitativos mais populares são os grupos focais, entrevistas pessoais e etnografia orientada para o mercado (ARNOULD; WALLENDORF, 1994). Dado que o método etnográfico envolve a observação participante do pesquisador, ele acaba sendo intencional e inevitavelmente um método intrusivo que impede uma observação discreta de consumidor. Grupos focais, nos quais se estabelece um contato face a face (CALDER, 1977) e entrevistas pessoais (THOMPSON, 1997) demandam menos tempo, são mais simples de se empregar e mais populares do que a etnografia. Contudo, sua intrusão, artificialidade e descontextualização podem apresentar deficiência ao serem comparadas aos métodos etnográficos.

Segundo relata Kozinets (2002), muitos antropólogos, sociólogos e pesquisadores de marketing escreveram sobre a necessidade de adaptar as técnicas de pesquisa etnográfica existentes para o uso em comunidades virtuais (ver, por exemplo, ESCOBAR et al., 1994; GROSSNICKLE; RASKIN, 2000; HAKKEN, 1999; KOZINETS, 1999; MILLER; SLATER, 2004), o que foi realizado pelo autor. Recentemente, segundo Kozinets (2014) muitos novos nomes foram dados ao método de etnografia online, incluindo "webnografia", "etnografia digital" e "ciberantropologia". No entanto, outros pesquisadores falharam em detalhar as etapas e procedimentos para se realizar uma netnografia.

Uma etnografia, segundo Kozinets (2014), seguiria a seguinte sequência: (i) fazer uma "entrada na cultura" (ii) recolher e analisar dados, (iii) assegurar sua interpretação confiável, (iv) realizar pesquisas éticas, e (v) proporcionar oportunidades para feedback dos membros da cultura. Já no caso da netnografia, na fase da 
"entrada na cultura" haveria duas etapas iniciais. Em primeiro lugar, os investigadores devem ter perguntas bem definidas e identificar os fóruns online específicos mais apropriados para seu interesse. Em segundo, eles devem aprender o máximo possível a respeito dos fóruns, grupos e participantes individuais que procuram entender.

Ao contrário das etnografias tradicionais, a identificação de comunidades relevantes através de uma pesquisa online é extremamente valiosa (KOZINETS, 2002). Os antigos grupos de Orkut, salas de batepapo virtuais ou grupos do Yahoo, acabaram evoluindo para outras formas de interação mais dinâmicas como o Facebook e o Whatsapp. Segundo Kozinets (2002), em geral, as comunidades devem ser escolhidas conforme os seguintes critérios: (i) que possuam um segmento de mercado ou um tópico relevante para a pesquisa; (ii) que apresentem maior tráfego de lançamentos e postagens; (iii) que apresentem maior número de indivíduos que 'postem' mensagens discretas; (iv) que possuam muitas informações detalhadas ou descritivas; (v) e que tenham mais interações entre os membros da comunidade relacionados com a pergunta de pesquisa. Quanto à coleta de dados, há duas fontes principais: os dados que os pesquisadores copiam diretamente das comunicações mediadas por computador e dos membros da comunidade online e os dados que os pesquisadores inscrevem sobre suas observações da comunidade e seus membros, interações e significados. Há uma distinta vantagem sobre os etnógrafos tradicionais, os netnógrafos conseguem transcrever automaticamente os documentos baixados ou copiados da internet. No entanto 0 problema maior reside no possível excesso de informações derivados da coleta.

Segundo Kozinets (2014), com a evolução da internet, as formas de interação proporcionaram uma gradativa migração de "laços fracos", em comunicações mediadas por e-mail, para laços mais fortes, caracterizados pelo "intercâmbio de muita informação ou pela presença de amizades pessoais íntimas" (MATEl; BALLROKEACH, 2003). Isto se reflete em plataformas como Instagram, Facebook e WhatsApp. Há também, dentro das mesmas plataformas de relacionamento, ou a medida que se aprofunda a busca de informações em mais de uma plataforma, uma progressão da coleta de informações de cunho inicialmente não social para atividades sociais cada vez mais associativas (KOZINETS,1999). Rheingold (1993, p. 5) define comunidades virtuais como sendo "agregações sociais que emergem da rede quando um número suficiente de pessoas [promove] discussões públicas por tempo suficiente, com suficiente sentimento humano, para formar redes de relacionamentos pessoais no ciberespaço".

Além desta definição, vale ressaltar que há um caráter real nas comunidades eletrônicas, já que "as pessoas que encontramos online não são virtuais. Elas são comunidades reais povoadas por pessoas reais" (KOZINETS, 1999, p. 21). Nas comunidades virtuais (RHEINGOLD, 1993), diferentes níveis de engajamento de seus membros podem ser objeto de classificação. Segundo Kozinets (2002), há quatro grupos principais: os Tourists (ou principiantes), com menores laços sociais e menor atividade; os Minglers (ou sociáveis), que têm fortes laços sociais, mas com um mínimo de interesse nas atividades do grupo; os Devotees (ou devotos), já com interesses de consumo fortes, mas pouco apego ao grupo; e por fim, os Insiders (ou confidentes), com fortes vínculos com o grupo online e alta atividade de consumo, tendendo a se manterem por longa data, sendo frequentemente referenciados como membros. Os grupos de menor engajamento podem migrar para os de maior.

A análise dos dados começa frequentemente concomitante com sua coleta. 0 netnógrafo deve contextualizar os dados, o que muitas vezes se revela mais desafiador no contexto online empobrecido da netnografia. Softwares podem auxiliar na categorização como o QSR, NVivo e Atlas Ti. Estas ferramentas podem promover o estabelecimento de ligação de dados, facilitando a construção da teoria (PACCAGNELLA 1997). No entanto, há uma perda da riqueza simbólica para se construir clareza. Talvez mais do que com a etnografia, algumas das interpretações mais úteis de dados netnográficos provém de sua riqueza contextual e podem ser resultado da penetração metafórica e simbólica de sua interpretação (THOMPSON, 1997).

Durante o curso de coleta e análise de dados netnográficos, os pesquisadores de mercado devem seguir determinado protocolo para que a pesquisa seja razoável ou digna de confiança. A netnografia baseia-se 
principalmente na observação de discurso (ARNOULD; WALLENDORF, 1994). Portanto, pode presumir-se que os informantes apresentem uma imagem mais cuidada e controlada, já que é mediada pela representação social (KOZINETS, 2014). No entanto, a netnografia parece perfeitamente adaptada à abordagem de Mead (1938), em que a unidade final de análise não é a pessoa, mas o comportamento ou 0 ato comunicativo. $O$ uso de dados online dessa maneira requer uma mudança radical da etnografia tradicional, que observa pessoas. A netnografia deve se concentrar na observação das comunicações e recontextualizá-las.

No entanto, a triangulação de dados netnográficos com dados coletados por outros métodos, como em entrevistas, grupos focais, pesquisas, ou etnografia tradicional, podem ser úteis se o pesquisador busca generalizar para grupos que não sejam as populações estudadas.

A questão ética é também de fundamental importância na condução de pesquisas, pois a netnografia usa informações que não são especificamente geradas para o pesquisador de marketing. Os consumidores que originalmente criaram os dados não necessariamente estariam de acordo com sua análise interpretativa. Caso um pesquisador engajado na netnografia agir de uma maneira considerada irresponsável e desrespeitosa pelos consumidores, estes podem danificar seu próprio meio e instrumento de pesquisa, podendo ocorrer uma supressão de informações, como relatado por Reid (1996). Exemplos de comportamento desrespeitosos seriam o de um homem se passar por mulher ou mentir sua idade para ser aceito em determinado grupo. Para tal, Kozinets (2002) estabelece quatro princípios éticos que devem ser seguidos pelo pesquisador: (i) $O$ investigador deve divulgar sua presença, afiliações e intenções para com os membros da comunidade durante qualquer pesquisa; (ii) os investigadores devem assegurar a confidencialidade e anonimato dos informantes; (iii) o pesquisador deve buscar e incorporar feedback e comentários de membros da comunidade online sendo pesquisado. 0 quarto procedimento é específico para o meio: (iv) o pesquisador deve tomar uma posição cautelosa sobre a questão do meio privado versus público.

Segundo Madden (2012), dois em cada três adultos online (63\%) afirmam que mantém um perfil em um site de redes sociais. No entanto, $58 \%$ dizem que seu perfil principal é definido como privado para que apenas os amigos possam vê-lo, o que denota a importância da questão da confidencialidade.

\section{Metodologia}

\section{Aplicando a Metodologia do Método Etnográfico}

Nesta seção, a netnografia é ilustrada como um método para explorar e analisar o fluxo de informações, interações entre membros, padrões de comportamento de comércio e divulgação de serviços online de uma comunidade de imigrantes brasileiros estabelecida na Austrália.

Para selecionar o grupo a ser estudado, foi utilizado um método semelhante ao proposto por Kozinets (2002) no qual as comunidades online devem ter, de preferência: (i) tópico ou grupo, com foco e segmento relevante à pesquisa, (ii) maior "tráfego" de lançamentos, (iii) um maior número de mensagens distintas, (iv) dados mais detalhados ou descritivamente ricos, e (v) mais interações entre os membros, do tipo exigido pela questão de pesquisa.

Para tal, foram acompanhados durante uma semana os seguintes grupos do Facebook: "Ajudando brasileiros em Buenos Aires", "Brasileiros em Danbury", "Brasileiros em Milão", "Brasileiros em Montreal", "Brasileiros em Perth", "Brasileiros em Queenstown", "Brasileiros na Dinamarca", "Brasileiro em Ibiza", "Londres para brasileiros", "Brasileiros em Sydney" e "Vancouver para Brasileiros". Foi escolhido o grupo "Brasileiros em Sydney", pois apresentou alto número de integrantes - sendo acima de 60.000 membros, intenso tráfego de postagens, variedade de interações intermediadas pelas postagens e quantidade significante de postagens relacionados à produtos, serviços e atividades comerciais.

Além da pesquisa netnográfica, utilizou-se os dados do relatório de pesquisa com 610 brasileiros morando na Austrália que destacam um perfil etário majoritariamente jovem, entre 20 e 40 anos, com predominância 
do gênero feminino. Estes indivíduos saíram do Brasil com visto de estudantes (em sua maioria), mas demonstraram fortes intenções em se estabelecer permanentemente no país. 0 clima e o estilo de vida (praiano) foram elementos cruciais para a decisão de imigração para a Austrália (CRUZ et al., 2016), conforme aponta o gráfico da Figura 1.

\section{Coleta e Apresentação dos Dados}

Primeiramente foi solicitada a permissão para entrada nos grupos. Após o aceite, iniciou-se a coleta de dados em si. Estipulou-se um intervalo de tempo que seria realizada a coleta das postagens. Através do comando "*" do Facebook é possível se fazer uma ordenação cronológica das informações postadas no grupo. Em seguida realizou-se a expansão dos comentários e posterior cópia e colagem em documento de word, para ser gerado uma versão final em $p d f$.

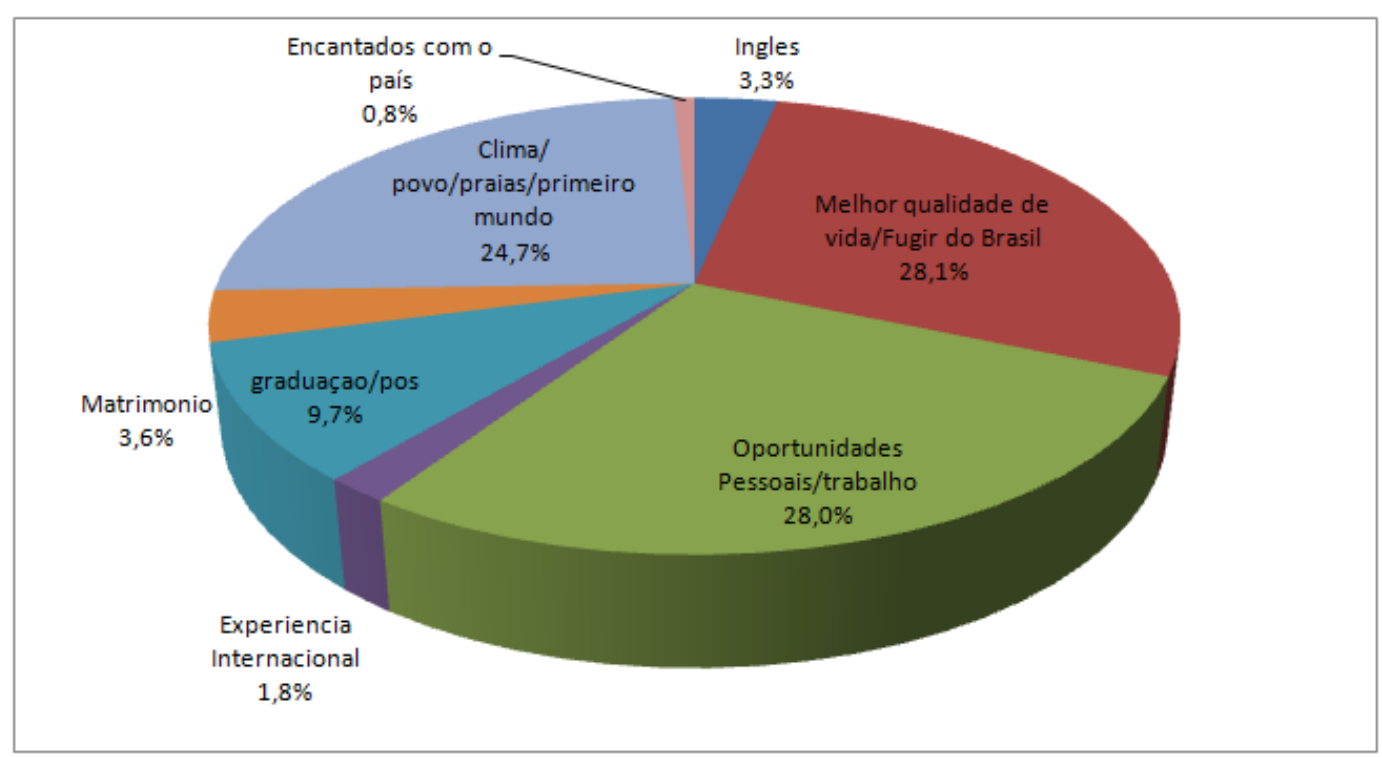

Figura 1: Por que a Austrália te atraiu?

Fonte: Cruz et al. (2016)

Para a análise de conteúdo utilizou-se o software Atlas Ti, sendo pré-determinadas algumas categorias de codificação. Foram contadas as postagens relacionadas a cada categoria, assim como extraídos trechos representativos de discursos de membros da comunidade que exibiam exemplos das categorias por meio do software.

No software de análise foram conduzidas duas etapas: (i) inserir as família de categorias que emergiram do campo e aquelas baseadas nos construtos retirados da literatura; (ii) definição dos código (itens do construto) e marcação das citações no documento final da coleta de dados. A árvore de pré-codificação das categorias está descrita na Figura 2.

Aúltima etapa da metodologia proposta consistiu na análise dos dados gerados pelos relatórios do software, à luz das teorias de senso de comunidade virtual, empreendedorismo étnico e transnacional. Lembrando que o foco do trabalho não é a avaliação da comunidade escolhida e sim a apresentação de uma aplicação da netnografia, optou-se por acompanhar a comunidade selecionada durante o período de 20/10/2016 à 27/10/2016. Algumas das categorias pré-codificadas não apresentaram postagens, o que pode indicar que sejam descartadas em trabalhos futuros, ou pesquisas em novas comunidades.

A Tabela 1 apresenta a contagem das postagens relativas às categorias no período de 20/10/2016 à 27/10/2016. Na seção discussão serão apresentados outros achados decorrentes da netnografia, 
confrontando-se com a literatura.

Baseado na investigação foi possível evidenciar que a comunidade virtual "Brasileiros em Sydney" é composta tanto de membros que vivem na cidade quanto de outros que habitam em diferentes lugares. Dos mais de 60.000 membros desta comunidade, pouco mais de 100 indivíduos possuem grande atividade em postagens constantes - os Insiders (ou confidentes), com fortes vínculos com o grupo online e alta atividade de consumo. Kozinets (2002) classifica ainda os Tourists (ou principiantes), como sendo os indivíduos com menor atividade no grupo e menores laços sociais, que apenas observam, e compõem a grande maioria do grupo. Estes indivíduos têm relação com a dimensão 'influência' de Blanchard e Markus (2004).

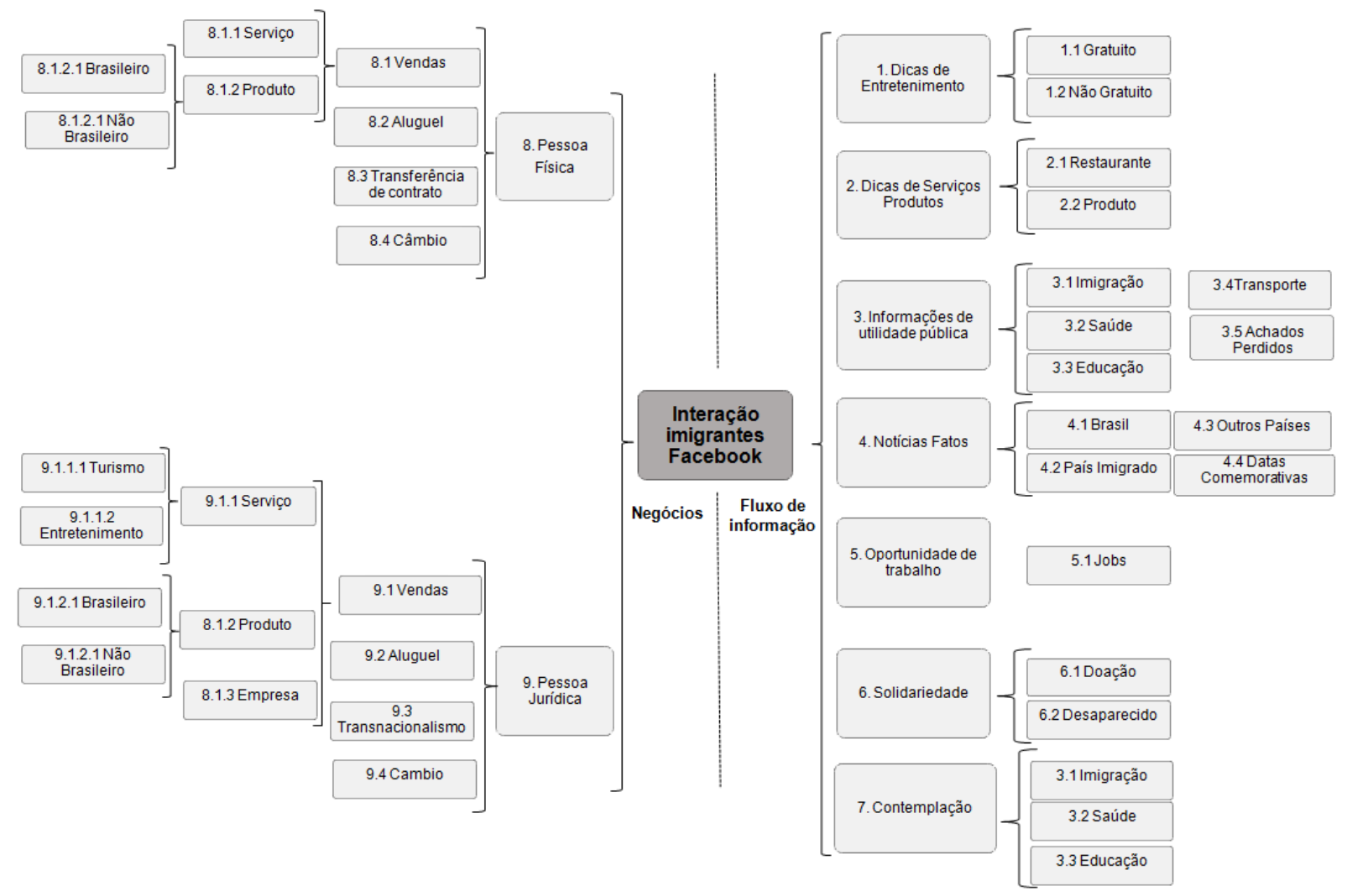

Figura 2: Pré-codificação das categorias

Fonte: elaboração própria

\begin{tabular}{l|l|l}
\hline Assuntos Tratados & Quantidade & Percentual \\
\hline Venda de Produtos Não Brasileiros & 77 & $21,4 \%$ \\
\hline Aluguel & 49 & $13,6 \%$ \\
\hline Dicas de Entretenimento & 35 & $9,7 \%$ \\
\hline Venda de Serviços & 37 & $10,3 \%$ \\
\hline Dicas de Imigração & 40 & $11,1 \%$
\end{tabular}


Roberto Pessoa de Queiroz Falcão, Andrea Ribeiro Carvalho Castro, Eduardo Picanço Cruz, Sabrina Sampaio Amaral

\begin{tabular}{l|l|l}
\hline Câmbio & 33 & $9,2 \%$ \\
\hline Oportunidade de Trabalho & 23 & $6,4 \%$ \\
\hline Texto, vídeo e fotos motivacionais & 16 & $4,4 \%$ \\
\hline Notícias_Fatos & 9 & $2,5 \%$ \\
\hline Dicas_Educação & 9 & $2,5 \%$ \\
\hline Solidariedade & 5 & $1,4 \%$ \\
\hline Compra de Produtos ou Serviços & 12 & $3,3 \%$ \\
\hline Venda de Produtos Brasileiros & 9 & $2,5 \%$ \\
\hline Transferência de Contrato & 2 & $0,6 \%$ \\
\hline Dicas_Transporte & 2 & $0,6 \%$ \\
\hline Dicas_Saúde & 2 & $0,6 \%$ \\
\hline TOTAL & 360 & $100,0 \%$ \\
\hline
\end{tabular}

Tabela 1: Resultado dos dias completos de 20/10 à 27/10/2016

Fonte: elaboração própria - dados da coleta.

\section{Discussão}

Por outro lado, não foi possível constatar a existência dos Minglers (ou sociáveis) ou dos Devotees (ou devotos). Seria necessário um maior tempo de envolvimento, por parte dos pesquisadores, assim como a complementação das observações com outros métodos de coleta, como entrevistas ou surveys envolvendo mais membros. Segundo (LI; BERNOFF, 2008, p. 45) a categoria dos espectadores abrange $48 \%$ dos norteamericanos adultos online, dois terços dos adultos japoneses online e dos habitantes das grandes cidades chinesas, e $37 \%$ dos europeus adultos online. Os autores apresentam o relatório do Forrester Research informando que $18 \%$ da população online adulta nos Estados Unidos, $10 \%$ dos adultos europeus e incríveis $38 \%$ dos sul-coreanos são criadores, as espinhas dorsais de muitas comunidades online.

É interessante perceber que a comunidade dos brasileiros em Sidney, reflete um grupo de imigrantes, composto em sua maioria de jovens que estão estudando ou vivendo temporariamente na cidade. Além do trabalho de Cruz et al. (2016), este perfil pode ser comprovado pela grande quantidade de venda de móveis usados, carros e geladeiras, semelhantes ao comércio que acontece em cidades universitárias, ou outras comunidades virtuais de estudantes. $21,4 \%$ das postagens de venda eram de produtos como celulares, carros, roupas, móveis, dentre outros. Apesar dos pesquisadores terem uma expectativa de colherem grande número de postagens de produtos étnicos como venda de alimentos e bebidas tradicionais do Brasil, esta categoria representou apenas $2,5 \%$ das postagens. O grupo também apresentou um considerável fluxo de postagens relativas à aluguel de quarto ou apartamentos $(13,6 \%)$, dicas de entretenimento $(9,7 \%)$, venda de serviços locais $(10,3 \%)$, dicas de imigração e vistos $(11,1 \%)$, e câmbio de moedas como dólar australiano ou americanos $(9,2 \%)$. 
Notou-se um senso de pertencimento de cultura brasileira - dimensão 'adesão' de Blanchard e Markus (2004), latente nos anúncios de festas brasileiras e algumas comidas comercializadas no grupo, como coxinhas, biscoitos e chocolates brasileiros. De acordo com Zhou (2004), como consumidores, os imigrantes preferem itens ligados ao país de origem, em termos de utilidade do bem ou da representação simbólica, relacionado à identidade de seu povo. Talvez devido à dificuldade de acesso, esse comércio informal de pequenos volumes de produtos da terra natal pelo Facebook seja uma forma de buscar este pertencimento. Além disso, se bem observado, essas postagens podem servir como embasamento para futuras importações regulares e comerciais desses produtos, ou seja, servir de germinação e teste de negócios étnicos.

Evidências também apontam para a maioria do grupo aparentando ser de classe média, e tendo como principal motivação o estudo de idioma e universitário (CRUZ et al., 2016). Contudo, não foram identificados postagens sobre trabalhos dentro de algum enclave étnico brasileiro, onde poderiam se comunicar na língua portuguesa. Ainda segundo CRUZ et al. (2016), o grupo de brasileiros na Austrália tem formação universitária. Foram identificadas postagens tanto em português como em inglês, o que evidencia que a língua inglesa não seria uma barreira. No tocante aos trabalhos locais, percebeu-se, em algumas postagens, uma preocupação com o valor que seria pago (se alto ou baixo), ou se valia à pena entrar em algum emprego, ou ficar apenas estudando.

Não foi observado no grupo o "transnacionalismo" ou empresários transnacionais. Embora ainda seja necessário investigar como os brasileiros se estabelecem como empresários na Austrália, 0 transnacionalismo normalmente ocorre quando as redes de negócios locais começam a funcionar, gerando fluxo de comércio que compense o retorno ao país de origem (BALTAR; ICART, 2013).
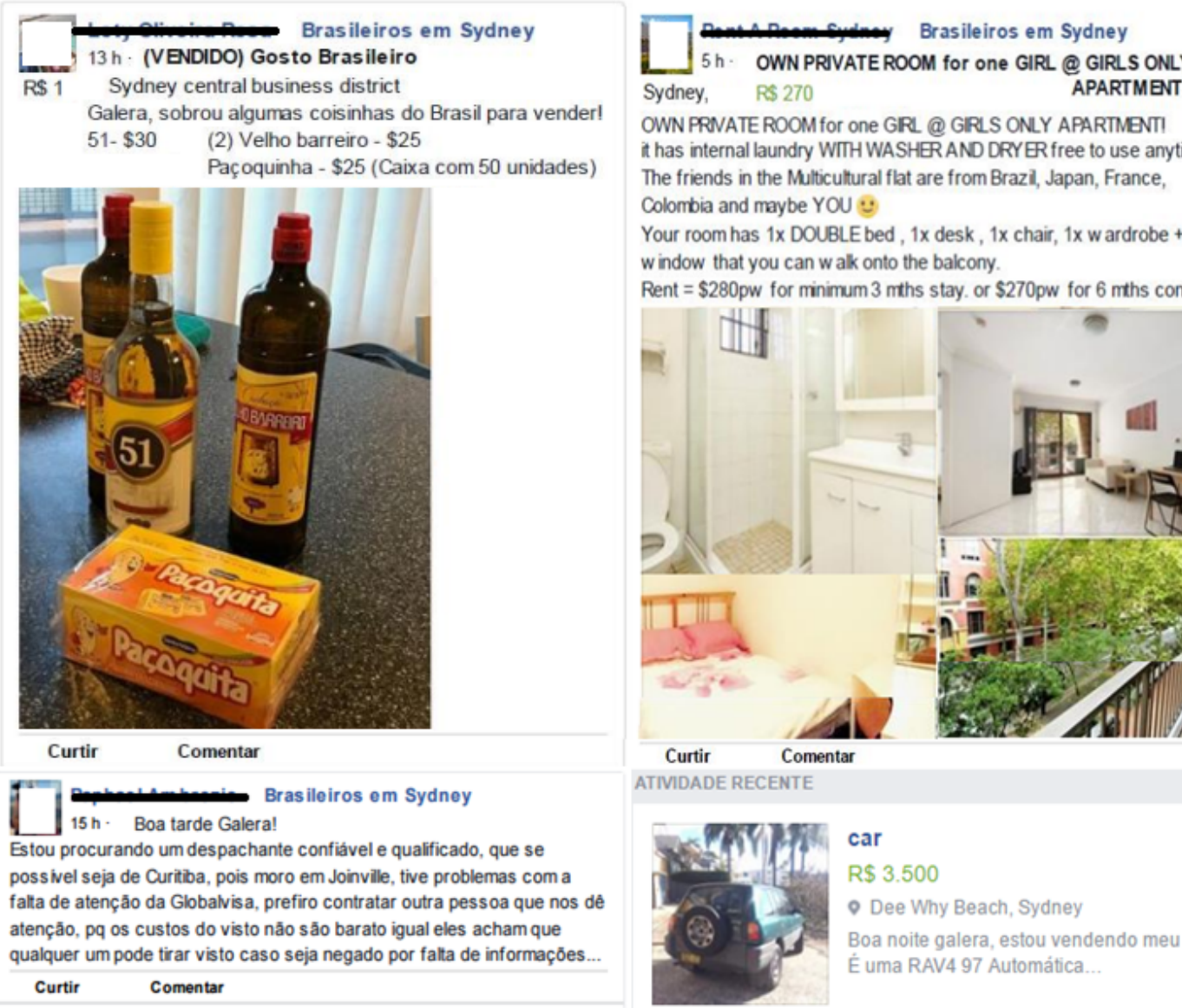

OWN PRNATEROOM for one GRL @ GIRLS ONLY APARTMENT! it has internal laundry WITH WASHERAND DRY $E$ R free to use anytime.

The friends in the Multicultural flat are from Brazil, Japan, France, Colombia and maybe YOU $:$

Your room has $1 \mathrm{x}$ DOUBLE bed, $1 \mathrm{x}$ desk , $1 \mathrm{x}$ chair, $1 \mathrm{x}$ w ardrobe $+\mathrm{a}$ big window that you can $w$ alk onto the balcony.

Rent $=\$ 280 \mathrm{pw}$ for minimum $3 \mathrm{mths}$ stay. or $\$ 270 \mathrm{pw}$ for $6 \mathrm{mths}$ contract.
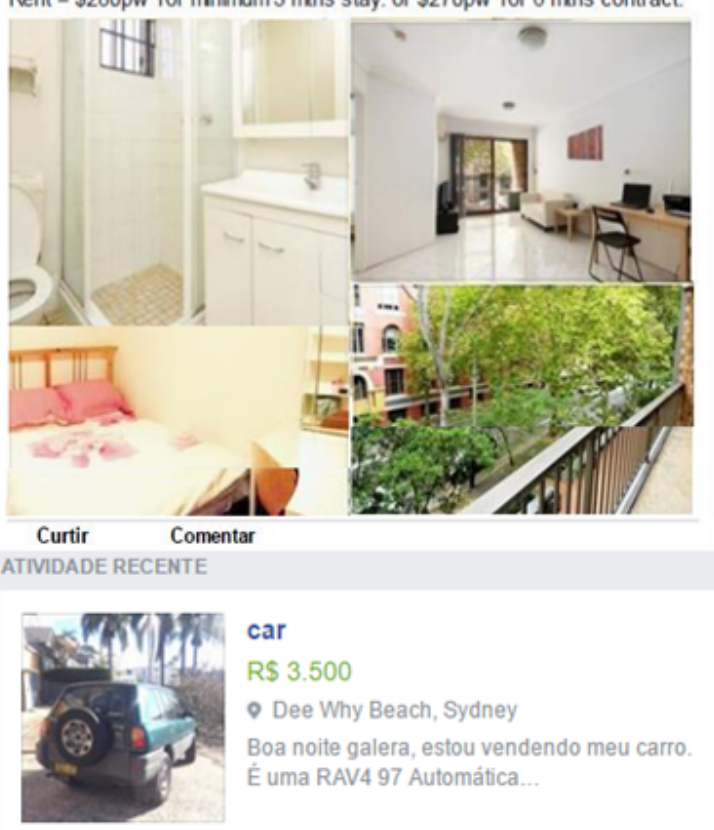

Figura 3: Exemplos de postagem

Fonte: elaboração própria - dados da coleta 
A comunidade, em grande parte, abarca imigrantes recentes, que ainda não estão totalmente integrados à sociedade australiana, e grupos de interessados em imigrar. Isto pode ser evidenciado pelas consideráveis postagens relativas às questões de imigração (11\%).

No tocante a esse item - histórias de imigração, as observações da comunidade corroboram as evidências de Fisher, Sonn e Bishop (2002) sobre o apego emocional e laços de união entre os membros. Algumas postagens enfocam a descrição das dificuldades e soluções para conseguir se manter legal ou tornar cidadão - uma das dicas recorrentes é fazer uma viagem de turismo na Nova Zelândia ou usar esse país que, segundo eles, oferece facilidades para o processo migratório.

Navarrete e Huerta (2006) destacam a relação da dimensão 'Integração e satisfação das necessidades' com experiências vividas, como por exemplo, a forma de enviar dinheiro para sua terra natal ou onde encontrar determinados ingredientes para seus pratos típicos. Somados os percentuais de postagens que envolviam dicas de câmbio, educação, transporte, saúde ou solidariedade em geral chega-se a 14,3\% do total.

Em relação à $4^{a}$ dimensão, 'conexão emocional compartilhada', foram evidenciadas dicas de passeio, festas eventos e até postagens convidando 'desconhecidos' para beber um drink no final do dia.

Kozinets (2014), afirma, baseado em McKenna e Seidman (2005), que os membros de comunidades virtuais, além de terem benefícios sociais diretos. Conforme constatado na comunidade dos brasileiros em Sidney, as comunidades online também têm efeitos poderosos sobre o senso de identidade dos indivíduos, o que corrobora também com os estudos de Blanchard e Markus (2004), Navarrete e Huerta (2006) e de McMillan e Chavis (1986).

No entanto há um limite entre o pertencimento e a promessa de inclusão e de uma autêntica experiência comunal e o foco de alguns anunciantes e comerciantes que identificam aquela comunidade online como um alvo comercial. Mesmo as pessoas físicas, membros aceitos da comunidade, aproveitam-se desse contexto comercial, vendendo seus móveis, carros, roupas, divulgando alugueis de quarto, dentre outras possibilidades.

Por fim, recorrendo ao trabalho de Pries (2004) que descreveu os tipos de migrantes internacionais, foram identificados na comunidade: o emigrante/imigrante, que tem a intenção de adotar o país de destino como sua nova pátria - presente nas diversas postagens pedindo informações e dicas ou reclamando do Brasil; 0 migrante de retorno, que pretende regressar à sua terra natal em um curto período de tempo - alunos que estão no programa Ciência sem Fronteiras, estudantes de MBA, Mestrado e Doutorado; e o transmigrante, que é ambíguo sobre a natureza e duração de sua migração - muitos brasileiros questionam se é fácil permanecer para sempre na Austrália, mesmo saindo do Brasil para estudar por um período limitado de tempo.

\section{Considerações Finais}

O método se apresentou como promissor, e traz possibilidades relativas a menores custos de implantação ao se buscar realizar investigações no exterior, de comunidades de imigrantes. Contudo, cabe apresentar a ressalva de Kozinets (2002) quanto às limitações do método, relativas ao grau de profundidade das investigações e pelo fato de não se estabelecer um contato face a face com os sujeitos de pesquisa. No entanto, o mesmo pode ser combinado com surveys e entrevistas online, que também complementariam a coleta dos dados remotamente, com custos inferiores à estratégias presenciais de coleta de dados. Todas estas técnicas funcionariam como triangulação de fontes. (DENZIN; LINCOLN, 1994).

No entanto, nada substitui entrevistas presenciais ou observação in loco de comunidades imigrantes. Para se almejar alegações mais gerais e adequadas de uma etnografia desse tipo, os pesquisadores necessitariam suplementar o trabalho netnográfico com vários outros tipos de investigação, tais como a observação de participantes in loco, pesquisas documentais nos sites de imigração australiana e entrevistas face a face com os principais membros e stakeholders da comunidade, visando-se entender 0 
comportamento dessa comunidade virtual no mundo real. Essa consideração também tem o caráter de sugestão de novos estudos.

Além dessa proposta estudos comparativos entre diferentes comunidades virtuais de imigrantes de uma mesma etnia poderiam iluminar questões não descobertas neste estudo preliminar, evidenciando a necessidade de novas categorias ou revelando outros padrões de comportamento.

Por fim, o trabalho traz uma implicação gerencial de proporcionar um método prático e acessível à investigações de cunho qualitativo sobre o comportamento das comunidades imigrantes no exterior, sendo bastante útil para pesquisadores que se envolvam com fenômenos observados em locais mais distantes: brasileiros no Japão, por exemplo.

\section{Referências}

ACHIDI, N. H.; PRIEM, R. L. Immigrant entrepreneurs, the ethnic enclave strategy, and venture performance. Journal of Management, v. 37, n. 3, p. 790-818, 2011.

ALIAGA-ISLA, R.; RIALP, A. Systematic review of immigrant entrepreneurship literature: previous findings and ways forward. Entrepreneurship \& Regional Development, v. 25, n. 9-10, p. 819-844, 2013.

APPADURAI, A. Disjuncture and difference in the global cultural economy. Theory, culture \& society, v. 7 , n. 2, p. 295-310, 1990.

ARNOULD, E. J.; THOMPSON, C. J. Consumer culture theory (CCT): Twenty years of research. Journal of consumer research, v. 31, n. 4, p. 868-882, 2005.

ARNOULD, E. J.; WALLENDORF, M. Market-oriented ethnography: interpretation building and marketing strategy formulation. Journal of marketing research, p. 484-504, 1994.

BALTAR, F.; ICART, I. B. Entrepreneurial gain, cultural similarity and transnational entrepreneurship. Global Networks, v. 13, n. 2, p. 200-220, 2013.

BARRETT, G. A.; JONES, T. P.; MCEVOY, D. Ethnic minority business: Theoretical discourse in Britain and North America. Urban studies, v. 33, n. 4-5, p. 783-809, 1996.

BENNETT, A. Subcultures or neo-tribes? Rethinking the relationship between youth, style and musical taste. Sociology, v. 33, n. 3, p. 599-617, 1999.

BLANCHARD, A. L.; MARKUS, M. L. The experienced sense of a virtual community: Characteristics and processes. ACM Sigmis Database, v. 35, n. 1, p. 64-79, 2004.

BLOEM, T. M. Impacts of Brazilian Business and Brazilian Immigrant Organizations in South Flórida. 2015. Doctoral dissertation, The University of Arizona.

BROWN, Stephen. Postmodern marketing research: no representation without taxation. Journal of the Market Research Society, v. 37, n. 3, p. 287-311, 1995.

BRUNEAU, M. Diasporas, transnational spaces and communities. Diaspora and transnationalism: concepts, theories and methods, p. 35-50, 2010.

BUTLER, K. D. Defining diaspora, refining a discourse. Diaspora: a journal of transnational studies, $v$. 10, n. 2, p. 189-219, 2001.

CALDER, B. J. Focus groups and the nature of qualitative marketing research. Journal of Marketing research, p. 353-364, 1977.

CASTELLS, M. The network society. Oxford: Blackwell, 1996.

CHRYSOSTOME, E.; LIN, X. Immigrant entrepreneurship: Scrutinizing a promising type of business venture. Thunderbird International Business Review, v. 52, n. 2, p. 77-82, 2010. 
COVA, B.; PACE, S. Brand community of convenience products: new forms of customer empowerment-the case "my Nutella The Community". European Journal of Marketing, v. 40, n. 9/10, p. 1087-1105, 2006.

CRUZ, E.P. et al. Relatório de Pesquisa Sobre Brasileiros na Austrália. UFF: Departamento de Empreendedorismo e Gestão. Recuperado em 28, abril, 2017, de http://uff.br/mpeinternacional/material/Relatorio_BrasilAustralia.pdf. 2016.

DENZIN, N. K.; LINCOLN, Y. S. Handbook of qualitative research. Sage publications, inc, 2008.

DRORI, I.; HONIG, B.; WRIGHT, M. Transnational entrepreneurship: An emergent field of study. Entrepreneurship Theory and Practice, v. 33, n. 5, p. 1001-1022, 2009.

ESCOBAR, A. et al. Welcome to Cyberia: Notes on the Anthropology of Cyberculture [and comments and reply]. Current anthropology, v. 35, n. 3, p. 211-231, 1994.

ESSERS, C.; BENSCHOP, Y.; DOOREWAARD, H. Female ethnicity: Understanding Muslim immigrant businesswomen in the Netherlands. Gender, Work \& Organization, v. 17, n. 3, p. 320-339, 2010.

FIRAT, A. F.; DHOLAKIA, N. Theoretical and philosophical implications of postmodern debates: some challenges to modern marketing. Marketing theory, v. 6, n. 2, p. 123-162, 2006. FIRAT, A. F.; VENKATESH, A. Liberatory postmodernism and the reenchantment of consumption. Journal of consumer research, $v$. 22, n. 3, p. 239-267, 1995.

FISHER, A. T.; SONN, C. C.; BISHOP, B. J. (Ed.). Psychological sense of community: Research, applications, and implications. Springer Science \& Business Media, 2002.

GROSSNICKLE, J.; RASKIN, O. The handbook of online marketing research: knowing your customer using the Net. McGraw-Hill Professional, 2000.

HAKKEN, D. Cyborgs@ cyberspace?: An ethnographer looks to the future. Psychology Press, 1999.

KIM, K. C.; HURH, W. M. Ethnic resources utilization of Korean immigrant entrepreneurs in the Chicago minority area. International Migration Review, p. 82-111, 1985.

KJELDGAARD, D.; ASKEGAARD, S. The glocalization of youth culture: The global youth segment as structures of common difference. Journal of consumer research, v. 33, n. 2, p. 231-247, 2006.

KLOOSTERMAN, R.; VAN DER LEUN, J.; RATH, J. Across the border: Immigrants' economic opportunities, social capital and informal business activities. Journal of ethnic and migration studies, v. 24, n. 2, p. 249268, 1998.

KOZINETS, R.V. On netnography: Initial reflections on consumer research investigations of cyberculture. ACR North American Advances, 1998.

KOZINETS, R. V. E-tribalized marketing?: The strategic implications of virtual communities of consumption. European Management Journal, v. 17, n. 3, p. 252-264, 1999.

KOZINETS, R. V. The field behind the screen: Using netnography for marketing research in online communities. Journal of marketing research, v. 39, n. 1, p. 61-72, 2002.

KOZINETS, R. V. Netnography 2.0. The Handbook of Qualitative Research Methods in Marketing. 2006.

KOZINETS, R. V. Inno-tribes: Star Trek as wikimedia. Consumer tribes, p. 194-211, 2007.

KOZINETS, R. V. Netnografia: realizando pesquisa etnográfica online. Penso Editora, 2014.

LIGHT, I. Global entrepreneurship and transnationalism. Handbook of research on ethnic minority entrepreneurship, p. 3-15, 2007.

LUEDICKE, M. K. Brand community under fire: The role of social environments for the HUMMER brand 
Pequenos negócios e fluxo de informações em comunidade de imigrantes: ensaio sobre aplicação da netnografia

community. Advances in Consumer Research, v. 33, p. 486-493, 2006.

MA, Z. et al. An overview of contemporary ethnic entrepreneurship studies: themes and relationships. International Journal of Entrepreneurial Behavior \& Research, v. 19, n. 1, p. 32-52, 2013.

MADDEN, M. Privacy management on social media sites. Pew Internet Report, p. 1-20, 2012.

MARGOLIS, M. L. Transnationalism and popular culture: the case of Brazilian immigrants in the United States. The Journal of Popular Culture, v. 29, n. 1, p. 29-41, 1995.

MARTES, A. C. B.; RODRIGUEZ, C. L. Afiliação religiosa e empreendedorismo étnico: o caso dos brasileiros nos Estados Unidos. Revista de Administração Contemporânea, v. 8, n. 3, p. 117-140, 2004.

MATEI, S.; BALL-ROKEACH, S. The Internet in the Communication Infrastructure of Urban Residential Communities: Macro-or Mesolinkage?. Journal of Communication, v. 53, n. 4, p. 642-657, 2003.

MCALEXANDER, J. H.; SCHOUTEN, J. W.; KOENIG, Harold F. Building brand community. Journal of marketing, v. 66, n. 1, p. 38-54, 2002.

MCKENNA, K.; SEIDMAN, G. You, me, and we: Interpersonal processes in electronic groups. The social net: Human behavior in cyberspace, p. 191-217, 2005.

MCKENNA, K. Y. A.; BARGH, J. A. Coming out in the age of the Internet: Identity" demarginalization" through virtual group participation. Journal of personality and social psychology, v. 75, n. 3, p. 681, 1998.

MCMILLAN, D. W.; CHAVIS, D. M. Sense of community: A definition and theory. Journal of community psychology, v. 14, n. 1, p. 6-23, 1986.

MEAD, G. H. The philosophy of the act (CW Morris, Ed.). 1938.

MIERA, F. Transnational strategies of Polish migrant entrepreneurs in trade and small business in Berlin. Journal of ethnic and migration studies, v. 34, n. 5, p. 753-770, 2008.

MILLER, D.; SLATER, D. Etnografia on e off-line: cibercafés em Trinidad. Horizontes antropológicos, v. 10, n. 21, p. 41-65, 2004.

NAVARRETE, C.; HUERTA, E. Building virtual bridges to home: The use of the Internet by transnational communities of immigrants, 2006.

NKONGOLO-BAKENDA, J. M.; CHRYSOSTOME, E. V. Engaging Diasporas as international entrepreneurs in developing countries: in search of determinants. Journal of International Entrepreneurship, v.11, n. 1, p. 30-64, 2013.

PACCAGNELLA, L. Getting the seats of your pants dirty: Strategies for ethnographic research on virtual communities. Journal of Computer-Mediated Communication, v. 3, n. 1, 1997.

PORTES, A.; ZHOU, M. Gaining the upper hand: Economic mobility among immigrant and domestic minorities. Ethnic and Racial Studies, v.15, n. 4, p. 491-522, 1992.

PRIES, L. Transnationalism and migration: new challenges for the social sciences and education. In: LUCHTENBERG, S. (Ed.), Migration, education and change. Londres: Routledge, 2004, p 15-39.

RATH, J.; KLOOSTERMAN, R. Outsiders' business: A critical review of research on immigrant entrepreneurship. International Migration Review, p. 657-681, 2000.

REID, E. Informed consent in the study of online communities: A reflection on the effects of computermediated social research. The Information Society, v. 12, n. 2, p.169-174, 1996.

RHEINGOLD, H. The Virtual Community: Homesteading on the Electronic Frontier.Reading, MA: AddisonWesley, 1993. 
RIDDLE, L. Diasporas: Exploring their development potential. ESR Review, v. 10, n. 2, p. 28, 2008.

SASAKI, E. M. Movimento dekassegui: a experiência migratória e identitária dos brasileiros descendentes de japoneses no Japão. In: Cenas do Brasil migrante. São Paulo: Boitempo, 1999, p. 243. -74

SASAKI, E. A imigração para o Japão. Estudos avançados, v. 20, n. 57, p. 99-117, 2006.

SCHAU, H. J.; MUÑIZ JR, A. M.; ARNOULD, E. J. How brand community practices create value. Journal of marketing, v. 73, n. 5, p. 30-51, 2009.

SCHOUTEN, J. W.; MCALEXANDER, J. H. Subcultures of consumption: An ethnography of the new bikers. Journal of consumer research, v. 22, n. 1, p. 43-61, 1995.

TEIXEIRA, C.; LO, L.; TRUELOVE, M. Immigrant entrepreneurship, institutional discrimination, and implications for public policy: a case study in Toronto. Environment and Planning C: Government and Policy, v. 25, n. 2, p. 176-193, 2007.

THOMPSON, C. J. Interpreting consumers: a hermeneutical framework for deriving marketing insights from the texts of consumers' consumption stories. Journal of marketing Research, p. 438-455, 1997.

VAN DELFT, H.; GORTER, C.; NIJKAMP, P. In search of ethnic entrepreneurship opportunities in the city: a comparative policy study. Environment and Planning C: Government and Policy, v. 18, n. 4, p. 429-451, 2000.

WALDINGER, R. D.; ALDRICH, H.; WARD, R. Ethnic entrepreneurs: Immigrant business in industrial societies. Sage Publications, Inc, 1990.

ZHOU, Min. The role of the enclave economy in immigrant adaptation and community building: The case of New York's Chinatown. Immigrant and minority entrepreneurship: building american communities, $p$. 37-60, 2004. 